\title{
Detection of micrometastases in peritoneal washings of gastric cancer patients by the reverse transcriptase polymerase chain reaction
}

\author{
Kimberly Moore Dalal ${ }^{1,2,3}$, Yanghee Woo ${ }^{1}$, Kaitlyn Kelly $^{1}$, Charles Galanis ${ }^{1}$, Mithat Gonen ${ }^{4}$, \\ YumAn Fong ${ }^{1}$, and DANIEl G. CoIT ${ }^{1}$ \\ ${ }^{1}$ Department of Surgery, Memorial Sloan-Kettering Cancer Center, 1275 York Avenue, New York, NY 10021, USA \\ ${ }^{2}$ Department of Surgery, David Grant U.S. Air Force Medical Center, Travis AFB, CA, USA \\ ${ }^{3}$ Department of Surgery, University of California at San Francisco, San Francisco, CA, USA \\ ${ }^{4}$ Department of Epidemiology and Biostatistics, Memorial Sloan-Kettering Cancer Center, New York, NY, USA
}

\begin{abstract}
Background. Gastric cancer patients with positive $(+)$ peritoneal cytology have a prognosis similar to stage IV patients. We studied the ability of quantitative reverse transcriptase polymerase chain reaction (RT-PCR) to detect peritoneal micrometastases in patients undergoing staging laparoscopy. Methods. Peritoneal washings were obtained prospectively from 34 patients with gastric adenocarcinoma undergoing staging laparoscopy and 6 patients undergoing laparoscopy for benign disease. Each sample underwent cytologic and RTPCR analysis for tumor markers: carcinoembryonic antigen (CEA), cytokeratin 20 (CK20), survivin, and MUC2. Markers were evaluated on the basis of their deviance from the ideal marker.

Results. Pathologic stages for the gastric cancer patients were: stage I, 9 (27\%); stage II, 7 (21\%); stage III, 15 (44\%); and stage IV, $3(9 \%)$. The four cytology (+) patients were: stage II, 1; stage III, 1; and stage IV, 2. Fifteen patients were RTPCR (+), including all cytology (+) patients. The optimal threshold for cycle amplification was 35 , based on a receiver operating characteristic curve. CEA had the smallest deviance.

Conclusion. RT-PCR using a panel of tumor markers, including CEA, detects (+) cytology. The clinical significance of "false-positive" overexpression of CEA, survivin, or CK20 but cytology (-) remains to be defined. RT-PCR could represent a more sensitive method than cytology for detection of subclinical peritoneal tumor dissemination; this may be useful in improving patient selection for operative management and clinical trials.
\end{abstract}

Key words Gastric cancer · Peritoneal washings · Cytology · Micrometastases $\cdot$ RT-PCR

Offprint requests to: D.G. Coit

Received: June 6, 2008 / Accepted: September 14, 2008

\section{Introduction}

Approximately 21500 new cases of gastric cancer are diagnosed annually in the United States, and 10880 patients are expected to succumb to this disease in 2008 [1]; the prognosis of these patients is poor (with a 5-year survival rate of $20 \%$ for all stages combined) and is primarily related to the extent of disease at presentation. The most frequent site of recurrence following R0 resection for gastric cancer is the peritoneum [2,3], resulting most likely from the intraperitoneal presence of free cancer cells shed from the serosal surface of the primary tumor [4]. Moreover, operative manipulation could result in dissemination of cancer cells, and this may be particularly true when suboptimal (D0) lymphadenectomy is performed. Cytologic examination of peritoneal washings at laparotomy has been used to detect these cells and predict the risk of recurrent disease [5, 6]. A close association between positive cytology and poor survival rate has been demonstrated $[5,7]$. However, intraperitoneal recurrence has been observed even in patients with negative cytology [8], suggesting a lack of sensitivity of conventional cytologic examination.

Reverse transcriptase polymerase chain reaction (RT-PCR) has been used to identify the presence of carcinoembryonic antigen (CEA) $[9,10]$ and cytokeratin 20 (CK20) mRNA in peritoneal washings of patients with gastric cancer [11, 12]. In one Japanese study, which demonstrated $80 \%$ sensitivity and $94 \%$ specificity rates of RT-PCR for CEA mRNA, the survival of CEA mRNA-positive patients was poor and approached that of cytology-positive patients [13]. Additionally, patients who were cytology-negative, but CEA mRNA-positive, had significantly poorer overall survival and diseasefree survival compared with CEA mRNA-negative patients [10].

Other tumor markers have been studied in peritoneal washings of gastric cancer patients. When RT-PCR for 
CK20 mRNA, with $63 \%$ sensitivity and $91 \%$ specificity rates, was combined with CEA mRNA, multivariate analysis identified the presence of both markers as a significant independent prognostic determinant [11]. Survivin is a protein, which, by RT-PCR, has a $66 \%$ sensitivity and $86 \%$ specificity [14]. Additionally, MUC2 has a specificity of $91 \%$ to $100 \%$ when used with other tumor markers [15]. The use of multiple tumor markers may optimize our ability to detect submicroscopic metastatic disease with a high degree of sensitivity and specificity.

The purpose of this study was to investigate the ability of a quantitative RT-PCR assay to detect mRNA of tumor markers overly expressed in peritoneal washings of patients undergoing laparoscopy for gastric cancer, to define the parameters to optimize RT-PCR, and to estimate the sensitivity, specificity, and false-positive and false-negative rates of peritoneal cancer cell detection by quantitative RT-PCR when compared to cytology as the gold standard.

\section{Methods}

\section{Patients}

From March to August 2006, peritoneal washings were obtained prospectively from 34 consecutive patients with gastric adenocarcinoma undergoing diagnostic laparoscopy for staging at Memorial Sloan-Kettering Cancer Center (MSKCC). Patients eligible for this pilot study were more than 18 years of age and presented to the Surgical Services at MSKCC with histologically confirmed gastric adenocarcinoma. Patients found to be potential candidates for surgical treatment based on history, physical examination, and conventional crosssectional body imaging were scheduled for laparoscopy. Patients were identified preoperatively, offered participation, and required to provide informed consent.

In addition, peritoneal washings were obtained from 6 patients undergoing laparoscopy for benign conditions (e.g., gallstones, hernia, and prophylactic risk-reducing bilateral salpingoophorectomy). These negative control research subjects were identified preoperatively by a member of the patient's treatment team at MSKCC. This study was conducted after MSKCC Institutional Review Board approval was gained.

\section{Cancer cell lines}

Established cell lines (BxPC3, HT-29) were obtained from the American Type Culture Collection (ATCC, Manassas, VA, USA). Gastric cell line OCUM was generously donated by Osaka City University Gradu- ate School of Medicine (Osaka, Japan). BxPC3 is a moderately differentiated pancreas cancer known to express MUC2 [16]. HT-29 is a colon cancer line which expresses CK20 [17] and survivin [18]. These cell lines were used as positive cancer controls for the RT-PCR assay. Cells were cultured as recommended and incubated at $37^{\circ} \mathrm{C}$ with $5 \% \mathrm{CO}_{2}$. RNA was isolated using the RNeasy Mini-Kit (Qiagen; Valencia, CA, USA) as described previously [19]. RT-PCR was performed with available RNA ( $\leq 2 \mu \mathrm{g})$ in a $100-\mu$ l reaction using random hexamer priming and TaqMan Reverse Transcription Reagents (Applied Biosystems; Foster City, CA, USA) on a Thermo Hybrid thermocycler (Waltham, MA, USA).

\section{Laparoscopic evaluation}

Patients underwent diagnostic laparoscopy in the standard fashion under general anesthesia as described previously [19]. Normal saline was introduced into the right upper abdomen, left upper abdomen, and pelvis and aspirated after gentle agitation but before manipulation of the primary or metastatic tumor. In all, three samples - from the right upper abdomen, left upper abdomen, and pelvis - were collected and divided into two parts: $30 \mathrm{ml}$ from each sample was sent to the pathology department for cytologic examination with conventional Papanicolaou staining. Additionally, $50 \mathrm{ml}$ was collected into a specimen cup and transported on ice to the laboratory for RNA isolation. During the laparoscopic examination, any visibly suspicious lesions were biopsied and sent for frozen section.

\section{Cytologic evaluation}

After retrieval, cytologic specimens were placed in Cytolyte fixative (Cytyc, Marlborough, MA, USA) and submitted to the cytology laboratory for evaluation. After centrifugation for $10 \mathrm{~min}$, the resulting cellular pellet underwent PreservCyte (Cytyc) fixation. Using the Thin Prep procedure, two slide preparations were made. The first was stained with a modified hematoxylin and eosin preparation and the second using the Papanicolaou method.

\section{RNA isolation}

Each of three peritoneal wash samples per patient was centrifuged at $800 \mathrm{rpm}$ for $5 \mathrm{~min}$ at $4{ }^{\circ} \mathrm{C}$. From each sample, $10 \mathrm{ml}$ of supernatant was removed for storage. The remaining specimens were centrifuged again at $800 \mathrm{rpm}$ for $5 \mathrm{~min}$ at $4{ }^{\circ} \mathrm{C}$. The three remaining pellets were combined and resuspended in $1 \mathrm{ml}$ of supernatant and centrifuged at $8000 \mathrm{~g}$ for $5 \mathrm{~min}$ at $4{ }^{\circ} \mathrm{C}$. The pellet was processed according to the RNeasy Mini-Kit 
(Qiagen) as described by the manufacturer and described previously [19].

\section{Real-time quantitative reverse transcriptase PCR (RT-PCR)}

Reverse transcription was performed using the TaqMan Universal Reverse Transcriptase Master Mix (Applied Biosystems). First, the amount of RNA isolated from the washings was assessed using spectrophotometry. While each reverse transcriptase reaction requires 1.5 to $2 \mu \mathrm{g}$ of RNA for optimal cDNA creation, most of these predominantly paucicellular specimens had less than $0.1 \mu \mathrm{g} / \mu \mathrm{l}$ of RNA. Therefore, we used the maximum volume of $38.5 \mu \mathrm{l}$ of RNA from each peritoneal wash sample which was amplified in a $100-\mu$ l reaction containing $1 \mathrm{uM}$ of each primer, $20 \mu \mathrm{l}$ of deoxynucleotide triphosphate, $25 \mathrm{mM} \mathrm{MgCl}, 10 \times$ Taq Buffer, random hexamers, RNAse inhibitor, and $2.5 \mu \mathrm{l}$ Multiscribe Reverse Transcriptase (Applied Biosystems). The samples were transferred to the Thermo Hybrid thermocycler at $25^{\circ} \mathrm{C}$ for $10 \mathrm{~min}, 48^{\circ} \mathrm{C}$ for $30 \mathrm{~min}$, and $95^{\circ} \mathrm{C}$ for $5 \mathrm{~min}$. The cDNA samples were stored at $-20^{\circ} \mathrm{C}$.

TaqMan Assays-on-Demand Gene Expression Assay primers for carcinoembryonic antigen (CEA), cytokeratin 20 (CK20), survivin, and MUC2 mRNA, as well as 18s rRNA were purchased from Applied Biosystems. Real-time quantitative RT-PCR was performed using the ABI-PRISM 7900 HT Sequence Detection System (Applied Biosystems). DNA was amplified in a $20-\mu \mathrm{l}$ reaction containing $1 \mu \mathrm{M}$ of the appropriate primer, $2 \mu \mathrm{l}$ cDNA, and TaqMan Gene Expression Master Mix including AmpliTaq Gold Polymerase. Each PCR reaction was subjected to initial setup of $30 \mathrm{~min}$ at $48^{\circ} \mathrm{C}$, $10 \mathrm{~min}$ at $95^{\circ} \mathrm{C}$, followed by 40 cycles at $95^{\circ} \mathrm{C}$ for $15 \mathrm{~s}$ and $60^{\circ} \mathrm{C}$ for $60 \mathrm{~s}$. Each sample was assayed in triplicate with positive PCR controls, which were the cell lines that overexpressed these tumor markers. The endogenous control gene, $18 s \mathrm{r} R N A$, was used to confirm the presence of mRNA in the peritoneal wash samples.

\section{Statistical analysis}

Patient data, including clinical characteristics and pathologic findings, were recorded and entered into a pro- spective database. Data for analysis included patient demographics, tumor pathologic characteristics, status of peritoneal cytology, and status of peritoneal tumor markers. The optimal threshold for cycle amplification was chosen using a receiver operating characteristic (ROC) curve [20] and previously described [19]. The ROC curve is constructed by plotting sensitivity and specificity pairs for tumor marker amplification cycles, resulting from varying the cutoff values over the range of results. The sensitivity and specificity of the tumor markers were estimated by using cytology as the gold standard, incorporating the information from negative controls as well as patients with malignancies. The "true positives" were defined as the patients who were found to have peritoneal metastases based on positive cytology or peritoneal metastases. Positive predictive value (PPV) and negative predictive value (NPV) of each marker were also computed. In addition, markers and their combinations were evaluated on the basis of their distance, or deviance, from the ideal marker (i.e., an ideal marker is $100 \%$ sensitive and specific).

\section{Results}

\section{Patient demographics}

From March through August 2006, 40 patients underwent laparoscopy and were entered into this pilot study; 34 patients had gastric cancer, and 6 had benign pathology. Patient demographic and clinicopathologic factors are shown in Tables 1-3. The median age of the cancer patients was 66 years (range, 40-81 years; Table 1). Fourteen patients $(41 \%)$ from the gastric cancer group were female. Within the gastric cancer group, $56 \%$ of patients $(n=19)$ underwent laparoscopy with biopsy while $44 \%$ underwent resection (distal subtotal gastrectomy, $n=12$; total gastrectomy, $n=3$ ).

Median age of the benign patients was 51 years (range, 41-68 years; Table 1); 83\% of the benign patients were female. Within the benign group, four patients underwent risk-reducing bilateral salpingooophorectomy, while the others underwent laparoscopic cholecystectomy or laparoscopic ventral hernia repair.

Among the gastric cancer patients, the tumor was located in the antrum in $47 \%(n=16)$ (Table 2$)$.

Table 1. Patient demographics

\begin{tabular}{|c|c|c|c|c|}
\hline & $\begin{array}{c}\text { Cancer patients } \\
n=34\end{array}$ & & $\begin{array}{l}\text { Benign patients } \\
\quad n=6\end{array}$ & \\
\hline Median age, years (range) & $66(40-81)$ & & $51(41-68)$ & \\
\hline Female sex & $14(41 \%)$ & & $5(83 \%)$ & \\
\hline \multirow[t]{3}{*}{ Procedure } & Laparoscopy and biopsy & 19 & Laparoscopic RRSO & 4 \\
\hline & Distal subtotal gastrectomy & 12 & Laparoscopic cholecystectomy & 1 \\
\hline & Total gastrectomy & 3 & Laparoscopic ventral hernia repair & 1 \\
\hline
\end{tabular}


Moderately differentiated adenocarcinoma occurred in $18 \%$ of the patients, while $71 \%$ had a moderately-poor to poorly differentiated cancer. A well-differentiated lesion was found in one patient. A T3 tumor was found in $53 \%(n=18)$ of the gastric cancer patients while $32 \%$ of the patients had a T2 tumor. T1 and T4 cancers were found in two and one patients, respectively. Another two patients $(6 \%)$ presented with an unknown $\mathrm{T}$ stage.

Pathologic stages for the gastric cancer patients were the following: stage IA, 1 (3\%); stage IB, $8(24 \%)$; stage II, 7 (21\%); stage IIIA, 13 (38\%); stage IIIB, 2 (6\%); and stage IV (peritoneal metastases), 3 (9\%; Table 3 ). Positive cytology was identified in 4 patients. Stages of their disease were stage II $(n=1)$, stage III $(n=1)$, and stage IV $(n=2)$. One patient with biopsy-proven peritoneal metastasis had negative cytology.

\section{Cytology}

The sensitivity and specificity of cytology to detect peritoneal disease in this cohort was $67 \%$ and $95 \%$, respectively. The PPV was 0.50 and NPV, 0.97 . There

Table 2. Pathologic variables

\begin{tabular}{lc}
\hline Variable & $n=34(\%)$ \\
\hline Location & \\
Gastroesophageal junction & $2(6)$ \\
Cardia & $5(15)$ \\
Lesser curve & $5(15)$ \\
Fundus & $2(6)$ \\
Antrum & $16(47)$ \\
Body & $2(6)$ \\
Entire & $2(6)$ \\
Differentiation & \\
Well to moderately well & $1(3)$ \\
Moderate & $6(18)$ \\
Moderately poor to poor & $24(71)$ \\
Not stated & $3(9)$ \\
T Stage & \\
Tx & $2(6)$ \\
T1 & $2(6)$ \\
T2 & $11(32)$ \\
T3 & $18(53)$ \\
T4 & $1(3)$ \\
\hline
\end{tabular}

were no cytology-positive patients in the benign patient group.

\section{Primer optimization and positive controls}

A total of four genes, CEA, CK20, survivin, and MUC2 were tested for expression in the cancer cell lines (Table 4). CEA and CK20 mRNA transcripts amplified at PCR cycle 22 in the OCUM and HT-29 cell lines, respectively. Survivin amplified in the HT-29 cell line at cycle 25 while MUC2 amplified in the BxPC3 cancer cell line at cycle 27. For each primer and cell line combination, the slopes of the PCR amplification ranged from -3.1 to -3.9 .

\section{Expression of $m R N A$ transcripts}

All 34 gastric cancer patients had amplifiable RNA, as detected by the amplification of the ribosomal $18 \mathrm{~s}$ subunit within 20 cycles.

ROC curve analysis was performed for tumor marker mRNA expression using data from all patients evaluated. Sensitivities and specificities were calculated based on the diagnosis of positive cytology made at laparoscopy. The point on the ROC curve that was closest to the ideal marker was achieved by using 35 cycles as the threshold (Fig. 1). Therefore, a tumor marker was deemed positive if 18s rRNA was amplified and if there was tumor marker cycle amplification within 35 cycles. Each potential tumor marker was evaluated separately.

Real-time RT-PCR using the TaqMan Assays-onDemand Gene Expression Assay allowed detection of CEA mRNA transcripts in eight patients within 35

Table 4. Optimization of gastric RT-PCR primers

\begin{tabular}{llcr}
\hline Primer & Cell line & Cycle & Slope \\
\hline CEA & OCUM & 22 & -3.20 \\
CK20 & HT29 & 22 & -3.13 \\
Survivin & HT29 & 25 & -3.94 \\
MUC2 & BxPC3 & 27 & -3.75 \\
\hline
\end{tabular}

Table 3. Stage and cytology

\begin{tabular}{lcccc}
\hline & $\begin{array}{c}\text { All cancer patients } \\
n=34(\%)\end{array}$ & $\begin{array}{c}\text { Positive cytology } \\
n=4\end{array}$ & $\begin{array}{c}\text { Any positive RT-PCR cytology/ } \\
n=15\end{array}$ & $\begin{array}{c}\text { positive RT-PCR } \\
n=11\end{array}$ \\
\hline Stage & & & & 0 \\
IA & $1(3)$ & 0 & 0 & 2 \\
IB & $8(24)$ & 0 & 2 & 2 \\
II & $7(21)$ & 1 & 3 & 1 \\
IIIA & $13(38)$ & 1 & 6 & 1 \\
IIIB & $2(6)$ & 0 & 3 & 5 \\
IV & $3(9)$ & 2 & 3 & \\
\hline
\end{tabular}



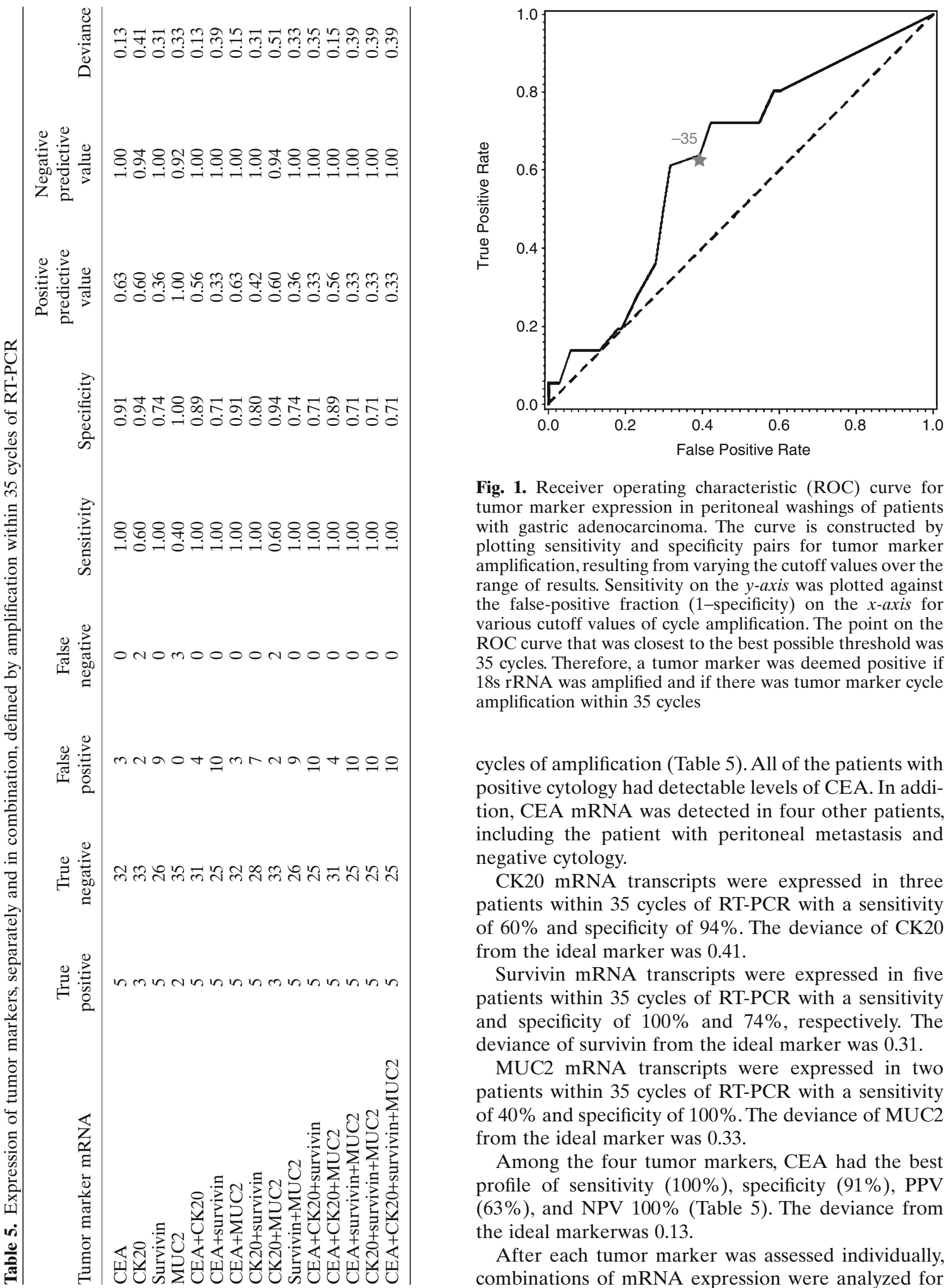

Fig. 1. Receiver operating characteristic (ROC) curve for tumor marker expression in peritoneal washings of patients with gastric adenocarcinoma. The curve is constructed by plotting sensitivity and specificity pairs for tumor marker amplification, resulting from varying the cutoff values over the range of results. Sensitivity on the $y$-axis was plotted against the false-positive fraction (1-specificity) on the $x$-axis for various cutoff values of cycle amplification. The point on the ROC curve that was closest to the best possible threshold was 35 cycles. Therefore, a tumor marker was deemed positive if 18s rRNA was amplified and if there was tumor marker cycle amplification within 35 cycles

cycles of amplification (Table 5). All of the patients with positive cytology had detectable levels of CEA. In addition, CEA mRNA was detected in four other patients, including the patient with peritoneal metastasis and negative cytology.

CK20 mRNA transcripts were expressed in three patients within 35 cycles of RT-PCR with a sensitivity of $60 \%$ and specificity of $94 \%$. The deviance of CK20 from the ideal marker was 0.41 .

Survivin mRNA transcripts were expressed in five patients within 35 cycles of RT-PCR with a sensitivity and specificity of $100 \%$ and $74 \%$, respectively. The deviance of survivin from the ideal marker was 0.31 .

MUC2 mRNA transcripts were expressed in two patients within 35 cycles of RT-PCR with a sensitivity of $40 \%$ and specificity of $100 \%$. The deviance of MUC2 from the ideal marker was 0.33 .

Among the four tumor markers, CEA had the best profile of sensitivity (100\%), specificity (91\%), PPV (63\%), and NPV 100\% (Table 5). The deviance from the ideal markerwas 0.13 .

After each tumor marker was assessed individually, combinations of mRNA expression were analyzed for 
sensitivity, specificity, PPV, NPV, and deviance (Table 5). Combinations of CEA+CK20, CEA+MUC2, and CEA+CK20+MUC2 had deviances of 0.13, 0.15, and 0.15 , respectively.

Overall, 15 patients had at least one marker positive by RT-PCR (Table 3). Eleven patients from stages IA through IV were cytology-negative but RT-PCRpositive, and they comprised the group of "falsepositives" with regard to RT-PCR when compared to the gold standard of cytology (Table 5). For the "falsepositive" gastric cancer patients, 3 patients expressed CEA mRNA transcripts; 9 patients were survivinpositive. CK20 and MUC2 mRNA transcripts were expressed in 2 and none of the "false-positive" gastric cancer patients, respectively.

Among the benign group which served as our negative controls, no patients were positive by RT-PCR for any of the four tumor markers.

\section{Discussion}

We have demonstrated that real-time RT-PCR can detect mRNA of tumor markers CEA, CK20, survivin, and MUC2 in peritoneal washings of patients undergoing laparoscopy for gastric cancer. Additionally, this pilot study defined the sensitivity, specificity, and falsepositive and false-negative rates of RT-PCR for the four tumor markers when compared to the gold standard, cytology. Moreover, these four tumor markers were evaluated separately and in combination. CEA alone had a sensitivity of $100 \%$, a specificity of $91 \%$, and a deviance of 0.13 from the ideal tumor marker. These findings are comparable to previous Japanese reports [13]. As CEA alone had the smallest deviance, detection of CK20, survivin, and MUC2 in combination with CEA did not appear to provide additional benefit.

The most frequent site of treatment failure following $\mathrm{R} 0$ resection for gastric cancer is the peritoneum [2,3], with a mean time to recurrence of 18 months [2]. To date, there are no effective therapies for peritoneal carcinomatosis, although there are randomized trials of intraperitoneal chemotherapy [21]. Therefore, detection of peritoneal disease prior to R0 resection is important for optimal treatment planning. Diagnostic laparoscopy with peritoneal washings and cytologic examination, accompanied by immunohistochemical staining with antibodies, has improved the sensitivity of detecting intraperitoneally shed tumor cells and predicting the risk of recurrent disease $[3,5,6]$. A close association between positive cytology and low survival rate has been demonstrated [5, 7, 22, 23]. Nevertheless, intraperitoneal recurrence has been observed even in patients with negative cytology [8], suggesting a lack of sensitivity of conventional cytologic examination.
Reverse transcriptase-PCR (RT-PCR) has been used to identify the presence of CEA $[9,10]$ and CK20 mRNA, the two most commonly studied markers, in patients with submucosal, muscularis propria, and subserosal gastric cancers in several Japanese studies [11, 12]. One report, by Kodera et al. [13], demonstrated that the survival of CEA mRNA-positive patients was poor and approached that of cytology-positive patients; recurrence was frequent among CEA mRNA-positive patients but rare for CEA mRNA-negative patients. Another report recently demonstrated that CEA positivity was an independent risk factor for cancer death and peritoneal recurrence after 5 years of follow up [24]; however, that study did not segregate out the incremental value of RT-PCR by analyzing cytology (-)/PCR (+) patients separately.

Analysis of multiple tumor markers may improve our ability to detect intraperitoneal submicroscopic metastatic disease with a high degree of sensitivity and specificity. RT-PCR for CK20 mRNA in our study had sensitivity and specificity rates of $60 \%$ and $94 \%$, respectively, rates which are comparable to previous studies [11]. When used with CEA, multivariate analysis has identified the presence of both markers as a significant independent prognostic determinant [11]. Our pilot study, however, did not demonstrate any benefit of additional markers, CK20, survivin, or MUC2 over CEA mRNA. In contrast to our findings, Mori et al. [25] recently showed that an RT-PCR-based microarray or immunocytochemical cytology including antibodies to CK20 and MUC2 had specificity and sensitivity rates that were better than conventional cytology.

While CEA had high sensitivity and specificity rates in our study, CK20, survivin, and MUC2 RT-PCR contributed to a large number of "false-positive" patients. Out of the 34 patients with gastric cancer, 15 patients had at least one marker positive by RT-PCR, 4 of whom were cytology (+)/RT-PCR (+), and 11 of whom were cytology $(-) /$ RT-PCR $(+)$. Nineteen patients were cytology $(-) /$ RT-PCR $(-)$. When we looked at the stages of disease, cytology status, and RT-PCR results, we identified 11 patients from all stages who could be considered to be false-positives. Therefore, if RT-PCR is truly identifying subclinical peritoneal disease, it potentially quadruples the number of cytology (+) patients among all gastric cancer patients. Moreover, when we looked at the 31 patients with stage I through III disease, 2 patients were cytology $(+) /$ RTPCR (+) and 17 patients were cytology (-)/RT-PCR $(-)$. Therefore, we identified 10 patients, or a yield of $35 \%$, who would be considered operative candidates after conventional cytology who are "false-positives". This underscores the potential importance of this technique in resectable patients. Discordance with negative 
cytology results may indicate that the tumor markers may increase the sensitivity of detection of disseminated disease in the peritoneal cavity, and this group of patients may represent an important population for study. The clinical significance of these "false-positives" will be elucidated by long-term follow up and analysis of recurrence and survival, as has been demonstrated with CEA mRNA [24].

Limitations of this pilot study included the small sample size and the logistical challenges of transporting the specimen from the patient to the laboratory within $1 \mathrm{~h}$. Transport time was minimized by improved communication with the operating room staff and laboratory personnel. Another important limitation was the inability to quantify the RNA with two separate spectrophotometers before converting the RNA to cDNA. From specimens that did undergo spectrophotometry, the concentrations of mRNA were roughly less than $0.1 \mu \mathrm{g} / \mu \mathrm{l}$. Two microliters of the RNA was used for reverse transcription, and $2 \mu \mathrm{l}$ of the cDNA template was used in the PCR reaction. The presence of mRNA was confirmed by amplification of the 18 s ribosomal subunit. In addition, the presence of tumor marker RNA was assessed by amplification of signal within 35 cycles of the PCR reaction. Although specimens may have amplified mRNA at various cycles of the PCR reaction, we could not quantify the tumor marker mRNA, because each sample did not begin with equal concentrations of mRNA. With the conclusion of this pilot study, we have begun to quantify mRNA with the use of a Nanodrop ND-1000 spectrophotometer (Wilmington, DE, USA). Additionally, there are benign causes for elevated CEA: smoking [26], inflammatory conditions including infections [27], inflammatory bowel disease [28], hypothyroidism [29], and pancreatitis and cirrhosis [30]. Levels can be raised transiently with radiation therapy [31]. Finally, assay variability can be a serious problem, and standardization for the volume of lavage fluid used and the RTPCR technique will be necessary if this method becomes widely practiced.

This pilot study is the first American study to confirm that real-time RT-PCR can detect mRNA of tumor markers overly expressed in peritoneal washings of patients undergoing laparoscopy for gastric cancer. Currently, our group is conducting a prospective, longitudinal study of 200 patients in order to define the predictors and clinical significance of a positive RTPCR result, with particular attention to CEA and CK20. An answer of clinical significance may not be fully available until adequate follow up of at least 2 years is obtained. Of interest will be the RT-PCR $(+) /$ cytology (-) patient undergoing curative resection. If a positive RT-PCR assay can identify a patient population at very high risk for early recurrence and death, this will clearly improve our therapeutic approach to patients with gastric cancer, as those patients may be unlikely to derive any meaningful benefit from resection. At the same time, however, there will invariably be a small number of RT-PCR-positive patients who may be cured. Whether this is due to the limitation of RT-PCR or the fact that a very small number of cells may be destroyed immunologically is unknown. Applying this diagnostic technique to staging laparoscopy could undoubtedly be informative, but avoiding surgery for all of the cytology (-), RT-PCR (+) patients could deprive this select population of the chances of cure, although a recent study at MSKCC, in which highly selected patients who had positive peritoneal cytology as their only evidence of metastatic disease received neoadjuvant chemotherapy and eventually underwent surgical resection with therapeutic intent, showed maintenance of a poor prognosis [23]. Our longitudinal study may provide some insight into improving the specificity of the RT-PCR examination. Nevertheless, the cost of conducting RT-PCR in these patients may provide the benefit of preventing the potential morbidity, operative mortality, subsequent decrease in quality of life, and additional costs that may result from an operation that will not result in a curative resection. Ultimately, this would then result in improved selection of patients for operative management, reduction in operative resection and morbidity in patients who will have poor survival, and establishment of the routine use of laparoscopy, cytology, and mRNA of tumor markers as part of the preoperative workup.

\section{Conclusion}

RT-PCR using a panel of tumor markers, including CEA, was comparable to cytology in terms of sensitivity, specificity, PPV, and NPV. The clinical significance of "false-positive" overexpression of CEA, CK20, or survivin in patients with negative cytology and no clinical evidence of peritoneal metastatic disease remains to be defined. RT-PCR could represent a more sensitive method than cytology for detection of subclinical peritoneal tumor dissemination; this may be useful in improving the selection of patients for operative management and clinical trials.

Acknowledgments We would like to acknowledge members of the Fong Laboratory, Yun Shin Chun, M.D., and David Eisenberg, M.D., as well as research study assistants Judy Fong and Maria Janakos. Kimberly Moore Dalal, M.D., had full access to all of the data in the study and takes responsibility for the integrity of the data and the accuracy of the data analysis. 


\section{References}

1. Jemal A, Siegel R, Ward E, Hao Y, Xu J, Murray T, et al. Cancer statistics, 2008. CA Cancer J Clin 2008;58:71-96.

2. Yoo $\mathrm{CH}$, Noh SH, Shin DW, Choi SH, Min JS. Recurrence following curative resection for gastric carcinoma. Br J Surg 2000; 87:236-42.

3. Benevolo M, Mottolese M, Cosimelli M, Tedesco M, Giannarelli $\mathrm{D}$, Vasselli S, et al. Diagnostic and prognostic value of peritoneal immunocytology in gastric cancer. J Clin Oncol 1998;16: 3406-11.

4. Koga S, Kaibara N, Itsuka Y, Kudo H, Kimura A, Hiraoka H. Prognostic significance of intraperitoneal free cancer cells in gastric cancer patients. J Cancer Res Clin Oncol 1984;108:236-8.

5. Kodera Y, Yamamura Y, Shimizu Y, Torii A, Hirai T, Yasui K, et al. Peritoneal washing cytology: prognostic value of positive findings in patients with gastric carcinoma undergoing a potentially curative resection. J Surg Oncol 1999;72:60-4.

6. Bonenkamp JJ, Songun I, Hermans J, van de Velde CJ. Prognostic value of positive cytology findings from abdominal washings in patients with gastric cancer. Br J Surg 1996;83:672-4.

7. Ribeiro U Jr, Gama-Rodrigues JJ, Safatle-Ribeiro AV, Bitelman B, Ibrahim RE, Ferreira MB, et al. Prognostic significance of intraperitoneal free cancer cells obtained by laparoscopic peritoneal lavage in patients with gastric cancer. J Gastrointest Surg 1998;2:244-9.

8. Abe S, Yoshimura H, Tabara H, Tachibana M, Monden N, Nakamura T, Nagaoka S. Curative resection of gastric cancer: limitation of peritoneal lavage cytology in predicting the outcome. J Surg Oncol 1995;59:226-9.

9. Kodera Y, Nakanishi H, Yamamura Y, Shimizu Y, Torii A, Hirai T, et al. Prognostic value and clinical implications of disseminated cancer cells in the peritoneal cavity detected by reverse transcriptase-polymerase chain reaction and cytology. Int J Cancer 1998;79:429-33.

10. Oyama K, Terashima M, Takagane A, Maesawa C. Prognostic significance of peritoneal minimal residual disease in gastric cancer detected by reverse transcription-polymerase chain reaction. Br J Surg 2004;91:435-43.

11. Kodera Y, Nakanishi H, Ito S, Yamamura Y, Fujiwara M, Koike $\mathrm{M}$, et al. Prognostic significance of intraperitoneal cancer cells in gastric carcinoma: detection of cytokeratin 20 mRNA in peritoneal washes, in addition to detection of carcinoembryonic antigen. Gastric Cancer 2005;8:142-8.

12. Marutsuka T, Shimada S, Shiomori K, Hayashi N, Yagi Y, Yamane T, et al. Mechanisms of peritoneal metastasis after operation for non-serosa-invasive gastric carcinoma: an ultrarapid detection system for intraperitoneal free cancer cells and a prophylactic strategy for peritoneal metastasis. Clin Cancer Res 2003;9:678-85.

13. Kodera Y, Nakanishi H, Ito S, Yamamura Y, Kanemitsu Y, Shimizu Y, et al. Quantitative detection of disseminated free cancer cells in peritoneal washes with real-time reverse transcriptase-polymerase chain reaction: a sensitive predictor of outcome for patients with gastric carcinoma. Ann Surg 2002;235: 499-506.

14. Wang ZN, Xu H, Jiang L, Zhou X, Lu C, Zhang X. Expression of survivin mRNA in peritoneal lavage fluid from patients with gastric carcinoma. Chin Med J (Engl) 2004;117:1210-7.

15. Mori K, Aoyagi K, Ueda T, Danjoh I, Tsubosa Y, Yanagihara K, et al. Highly specific marker genes for detecting minimal gastric cancer cells in cytology negative peritoneal washings. Biochem Biophys Res Commun 2004;313:931-7.

16. Yamada N, Hamada T, Goto M, Tsutsumida H, Higashi M, Nomoto $\mathrm{M}$, et al. MUC2 expression is regulated by histone $\mathrm{H} 3$ modification and DNA methylation in pancreatic cancer. Int $\mathrm{J}$ Cancer 2006;119:1850-7.

17. Wharton RQ, Jonas SK, Glover C, Khan ZA, Klokouzas A, Quinn $\mathrm{H}$, et al. Increased detection of circulating tumor cells in the blood of colorectal carcinoma patients using two reverse transcription-PCR assays and multiple blood samples. Clin Cancer Res 1999;5:4158-63.

18. Zhang T, Otevrel T, Gao Z, Gao Z, Ehrlich SM, Fields JZ, et al. Evidence that APC regulates survivin expression: a possible mechanism contributing to the stem cell origin of colon cancer. Cancer Res 2001;61:8664-7.

19. Dalal KM, Woo Y, Galanis C, Gonen M, Tang L, Allen P, et al. Detection of micrometastases in peritoneal washings of pancreatic cancer patients by the reverse transcriptase polymerase chain reaction. J Gastrointest Surg 2007;11:1598-606.

20. Zweigh MH, Campbell G. Receiver-operating characteristics (ROC) plots, a fundamental evaluation tool in clinical medicine. Clin Chem 1993;39:561-77.

21. Yan TD, Black D, Sugarbaker PH, Zhu J, Yonemura Y, Petrou G, et al. A systematic review and meta-analysis of the randomized controlled trials on adjuvant intraperitoneal chemotherapy for resectable gastric cancer. Ann Surg Oncol 2007;14:270213.

22. Bentrem D, Wilton A, Mazumdar M, Brennan M, Coit D. The value of peritoneal cytology as a preoperative predictor in patients with gastric carcinoma undergoing a curative resection. Ann Surg Oncol 2005;12:347-53.

23. Gold JS, Jaques D, Bentrem DJ, Shah MA, Tang LH, Brennan MF, et al. Outcome of patients with known metastatic gastric cancer undergoing resection with therapeutic intent. Ann Surg Oncol 2007; 14:365-72.

24. Kodera Y, Nakanishi H, Ito S, Mochizuki Y, Ohashi N, Yamamura Y, et al. Prognostic significance of intraperitoneal cancer cells in gastric carcinoma: analysis of real time reverse transcriptase-polymerase chain reaction after 5 years of followup. J Am Coll Surg 2006;202:231-6.

25. Mori K, Suzuki T, Aozaki H, Nakanishi H, Ueda T, Matsuno Y, et al. Detection of minimal gastric cancer cells in peritoneal washings by focused microarray analysis with multiple markers: clinical implications. Ann Surg Oncol 2007;14:1694-702.

26. Chu TM. Current status of carcinoembryonic antigen assay. Semin Nucl Med 1975;5:255-62.

27. Paavonen J, Miettinen A, Heinonen PK, Aaran RK, Teisala K, Aine R, et al. Serum CA 125 in acute pelvic inflammatory disease. Br J Obstet Gynaecol 1989;96:574-9.

28. Gardner RC, Fienerman AE, Kantrowitz PA, Gottblatt S, Loewenstein MS, Zamcheck N. Serial carcinoembryonic antigen (CEA) blood levels in patients with ulcerative colitis. Am J Dig Dis 1978;23:129-33.

29. Amino N, Kuro R, Yabu Y, Takai SI, Kawashima M, Morimoto $\mathrm{S}$, et al. Elevated levels of circulating carcinoembryonic antigen in hypothyroidism. J Clin Endocrinol Metab 1981;52:457-62.

30. Loewenstein MS, Zamcheck N. Carcinoembryonic antigen (CEA) levels in benign gastrointestinal disease states. Cancer 1978;42: 1412-8.

31. Jakobsson PA, Wahren B. Elevated plasma CEA during radiotherapy for glottic carcinoma of the larynx. Can J Otolaryngol $1975 ; 4: 46-50$. 